\title{
EXPERIMENTAL INVESTIGATION OF ACOUSTIC AND CAVITATION PROCESSES GENERATED BY THE LASER - LITHOTRIPSY
}

\author{
B.C. IHLER and A. HIRTH \\ Institut Franco-Allemand de Recherches de Saint-Louis-ISL, 5 rue de l'Industrie, \\ F-68300 Saint-Louis cedex, France
}

\begin{abstract}
Up to now, the fragmentation processes occurring at the Laser-Lithotripsy are not well enough investigated. The main emphasis of this work lies on the visualization and the explanation of acoustic and cavitation processes taking place during the time after plasma ignition on the stone surface generated by a dye laser.
\end{abstract}

Introduction:

The Laser-Lithotripsy is, in addition to the Extracorporal- Shockwave-Lithotripsy (ESWL), a less invasive method of destroying urinary-, gall- and salivary stones in the human body. The pulsed laser energy, guided by an endoscope, is transmitted via a small optical fiber $(200 \mu \mathrm{m})$ to the surface of the stone.

There are several laser-systems for this special application. These systems differ in the pulse length, which causes, related to other application specific parameters, basically different fragmentation processes. In that case, one has to distinguish between two groups. The pulse length of the first group lies in the nanosecond range only represented by the Q-switched Nd:YAG laser. Due to the very high power density at the distal end of the fiber, a plasma will be generated directly in the physiological liquid in front of the stone by a Laser-Induced Breakdown (LIB). The second group, reaching from some 100ns to some $1 \mu$ s, includes both dye lasers and solid-state lasers like the Alexandrite and the Titan:Sapphire laser. Here we have other plasma ignition mechanisms. Due to the lower power density, the plasma formation happens on the stone surface, mainly caused by absorption processes and is therefore strongly dependend on the characteristic of the stone.

At present the most used systems belong to the second group which allows a much safer energy transmission via the small fiber than with the Q-switched Nd:YAG laser, working close to the destruction threshold of quartz.

The results of the discussed investigations using a specially developed close-coupled 12"-dye laser $\left(\mathrm{T}_{F W H M}=1.5 \mu \mathrm{s}, \mathrm{E}_{\max }=1 \mathrm{~J}, \lambda=504 \mathrm{~nm}\right.$ - Coumarin 504) are representative for the second group.

Experimental Arrangements:

Different experimental arrangements has been used for the visualization of the single fragmentation processes. The spreading of shockwaves can be studied by using a Schlieren-set-up [1] that is shown in fig.1. The optical space filter offers a 2D-Schlieren sensitivity. With this arrangement even weak refraction gradients can be visualized. The exposure is done by a frequency-doubled Q-switched Nd:YAG laser, The exposure time of $\mathbf{T}_{\exp }=20 \mathrm{~ns}$ yields sharp images of shockwaves, which spread in the direct surrounding of the emission center in water with $\mathrm{v}_{s} \approx 1620 \mathrm{~m} / \mathrm{s}(\mathrm{P}=1 \mathrm{kbar}, \mathrm{T}=20$ degree $\mathrm{C})$ [2]. This corresponds to an unsharpness of $\mathrm{d} \approx 32 \mu \mathrm{m}$. For studying the bubble growth and the following cavitation processes, the set-up in fig. 2 offers better results. The illumination is done by a $600 \mu \mathrm{m}$ fiber from the same direction the photo has been taken. A diffuse reflecting aluminum plate in the background offers, on the one hand, a regular illuminated photograph, and, on the other hand, a view into the bubble. The arrangements in fig. 1 and fig. 2 offer only single photographs taken at different shots with different delay times. In order to study the process dynamics at one fragmentation a high-speed-camera has to be installed. Fig. 3 shows the experimental arrangement for the cinematography. To record the whole process which lasts about a millisecond, a drum camera (Strobodrum) with 11,000 frames/s is used. The exposure is done by an Ar:Ion laser. The exposure time is set by an intra-cavity acousto-optical shutter ( $t_{\text {exp }}=700 \mathrm{~ns}$ offers an unsharpness 
of $\left.\mathrm{d} \leq 98 \mu \mathrm{m} ; \mathrm{v}_{b u b b . w a l l} \leq 140 \mathrm{~m} / \mathrm{s}\right)$. [3]

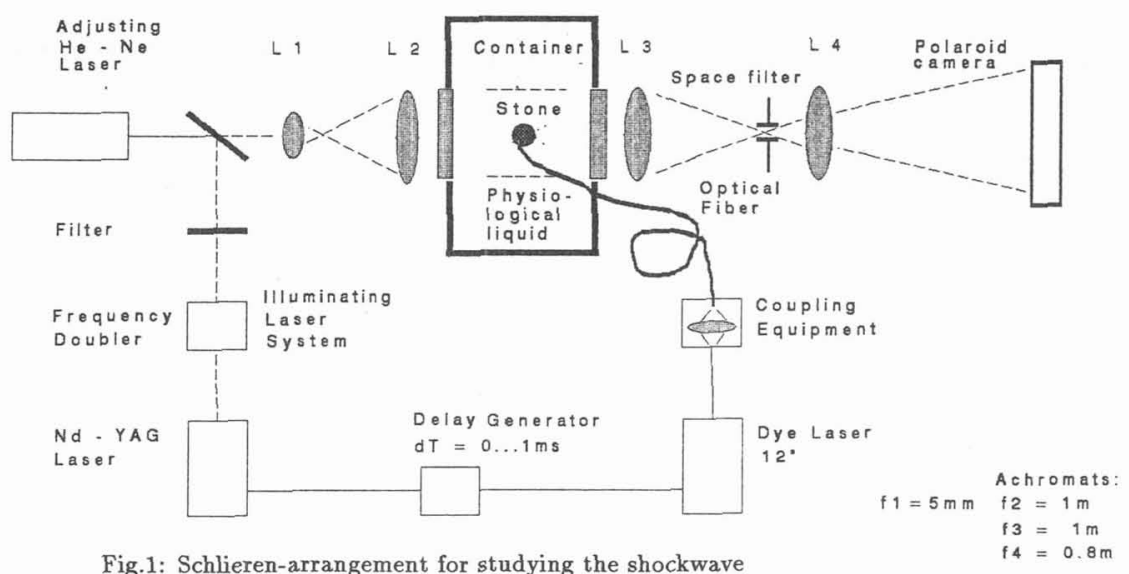

Fig.1: Schlieren-arrangement for studying the shockwave spreading at the plasma expansion phase

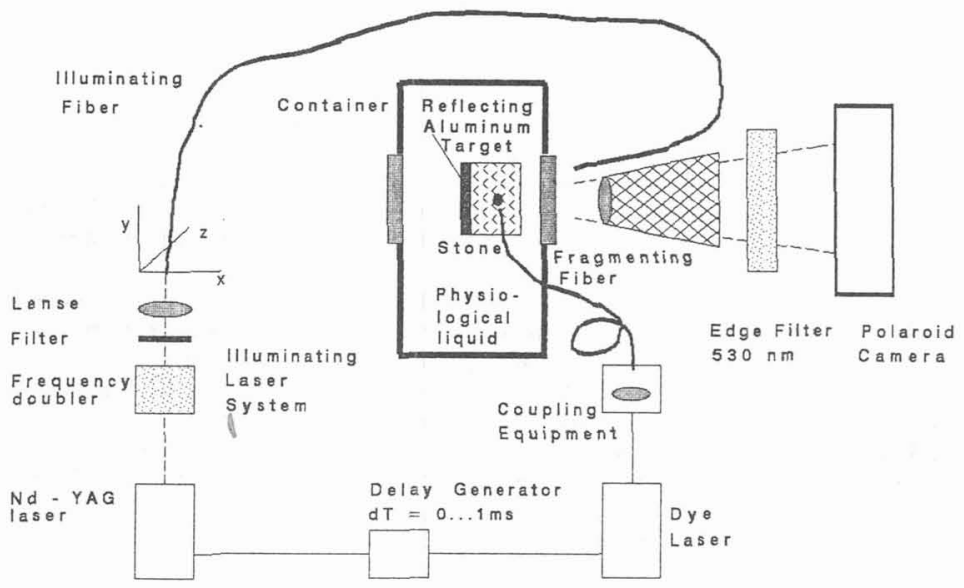

Fig.2: Experimental set-up for the investigation of the bubble expansion and collapse phase

Results:

Within the plasma expansion phase, that starts dependent on the pulse energy after some 100ns [4], there are two main processes. At first, the emission of the so-called primary shockwave and, secondly, the bubble formation. There is no worth mentioning fragmentation in form of hurled stone particles at the impact of the primary shockwave, which spreads hemispherically into the space above the stone (fig.4 series 1 No.1-4). However, it is likely that first micro-cracks will appear. Fragmentation particles are normally hurled to the stone surface in direction of the fiber not before the bubble collapses (No.5; the bubble has already disappeared). Within the bubble expansion phase, the bubble shape is nonhemispherical. The elongation normal to the solid surface is caused by the slowed down bubble growth close to this surface [3] (fig. 4 series 3 with $R^{\prime}=R_{\text {normal }} / R_{\text {parallel }}: R_{1}^{\prime}=1.02, R_{2}^{\prime}=1.12, R_{3}^{\prime}=1.15$ ). The maximum bubble radius at the gallstone fragmentation, shown in series 2 and series 3 , lies between $R_{\max }=3.5-4.5 \mathrm{~mm}$ and depends on the process $\left(E_{\text {fiber }}=150 \mathrm{~mJ} ; \mathrm{d}_{\text {fiber }}=400 \mu \mathrm{m} ; \mathrm{D}=79.6 \mathrm{MW} / \mathrm{cm}^{2}\right)$. After reaching its maximum size, the bubble dynamics can be considered as a special case of the classical cavitation theory for collapsing cavities near solid boundaries with $\gamma=0\left(\gamma=s / R_{m a x}\right.$ - ratio of distance between emission center and solid surface $s$ and $\max$. bubble radius $\left.R_{\max }\right)$. [3] 


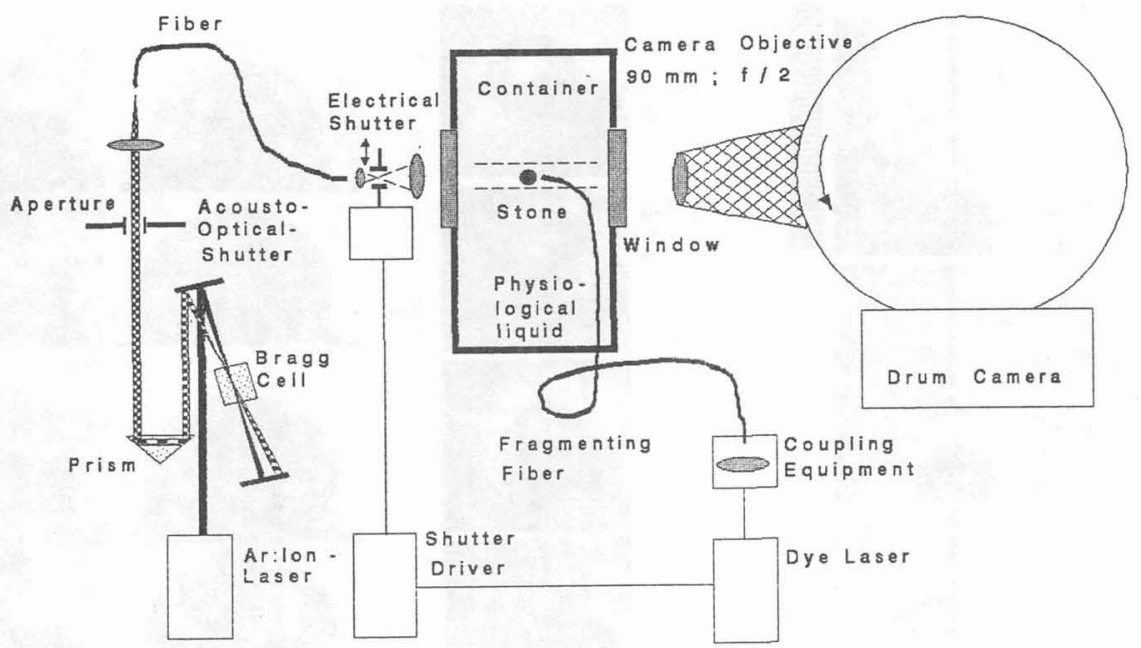

Fig.3: Experimental arrangement for cinematography with an intracavity acousto-optical shutter for setting the exposure time.

Naudé and Ellis [5], Plesset and Chapman [6], Blake, Taib and Doherty [7], Vogel, Lauterborn, Timm [3] and others have investigated cavitation processes with $\gamma>0$ above nearly infinite plane solid surfaces. In this special case of Laser-Lithotripsy, there is a cavitation process directly on the stone-surface $(\gamma=0)$, which has an extension in the order of the maximum cavitation bubble size. Stones in the human body are either surrounded by tissue, like in the urethra, or lie relatively free. The latter is simulated in above experimental arrangements, where the stones are carried either by a holder or positioned free on a target.

According to the differences in the experimental set-up, there is another bubble dynamics for the collapse phase. The plasma, which is directly ignited on the stone surface, is able to expand only into the hemisphere above the stone. The bubble formation is initiated by the explosive plasma expansion and therefore the bubble growth is also directed into this hemisphere. Due to the strong surface tension, the bubble adheres to the stone and their underside gets plane, shown in No.1 of series 2 , as soon as the bubble diameter gets bigger than the stone dimensions. It is known from Rayleigh [8] that the collapse time for a bubble is proportional to its radius. In the model the quasi-hemispherical bubble can be regarded as a formation of a lot of single bubbles with different radii. The bubbles with the smallest radii sited nearby the underside collapse first, so that the cavitation bubble gets the shape of a mushroom (mushroom cloud) (fig.4 series 2 No.3,4,5 and series 3 No.5,6). Due to similar dimensions of bubble and stone, the liquid flow is in the beginning of the collapse phase nearly unhindered. Later on it is limited mainly by the adhesion of the bubble underside on the stone.

A liquid jet against the solid wall, which is normally formed out for $\gamma \rightarrow 0[3,7]$, has not been observed. There is, furthermore, a liquid flow pointed to the opposite direction, versus the fiber (series 1 No.5, series 2 No.6,7 and series 3 No.7-14). As a consequence of these processes, the fiber, which is only guided but not fixed, recoils (series 3 No.10-14). Qualitative pressure measurements with a PVDF-pressure sensor showed that a strong shockwave is emitted within the collapse phase, followed by fragmentation particles which are hurled away from the stone surface in direction of the fiber. After the first collapse the bubble recoils. The measured pressure signal for the second collapse was very small. Reasons for this can be the smaller bubble size and the shape of the second bubble, which has a irregular, starlike appearance (series 2 No.6,7). Due to the latter a uniform collapse can't be observed.

\section{Conclusion:}

For the special case of Laser - Lithotripsy with long-pulse lasers in the microsecond range, where the bubble collapse takes place on a solid surface (stone) in the dimensions of the maximum bubble size, the bubble dynamics differs basically from the classical cavitation theory for collapsing cavities near solid bounderies. The investigations mentioned above have shown, that the real fragmentation occurs not before the first bubble collapse. This collapse takes place in the shape of a collapsing mushroom. A liquid jet against the solid surface could not be observed. The absorption dependent processes show great fluctuation related to the maximum of the bubble size, the collapse time depending on the latter and on the pressure emission. Further investigations with higher time-resolution will be done to verify the discussed results. 


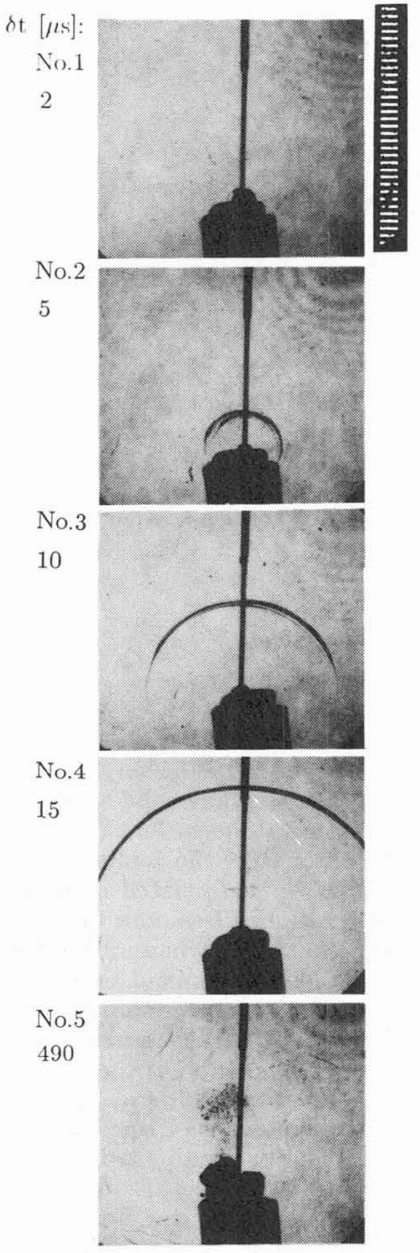

Series 1

arrangement of fig. 1
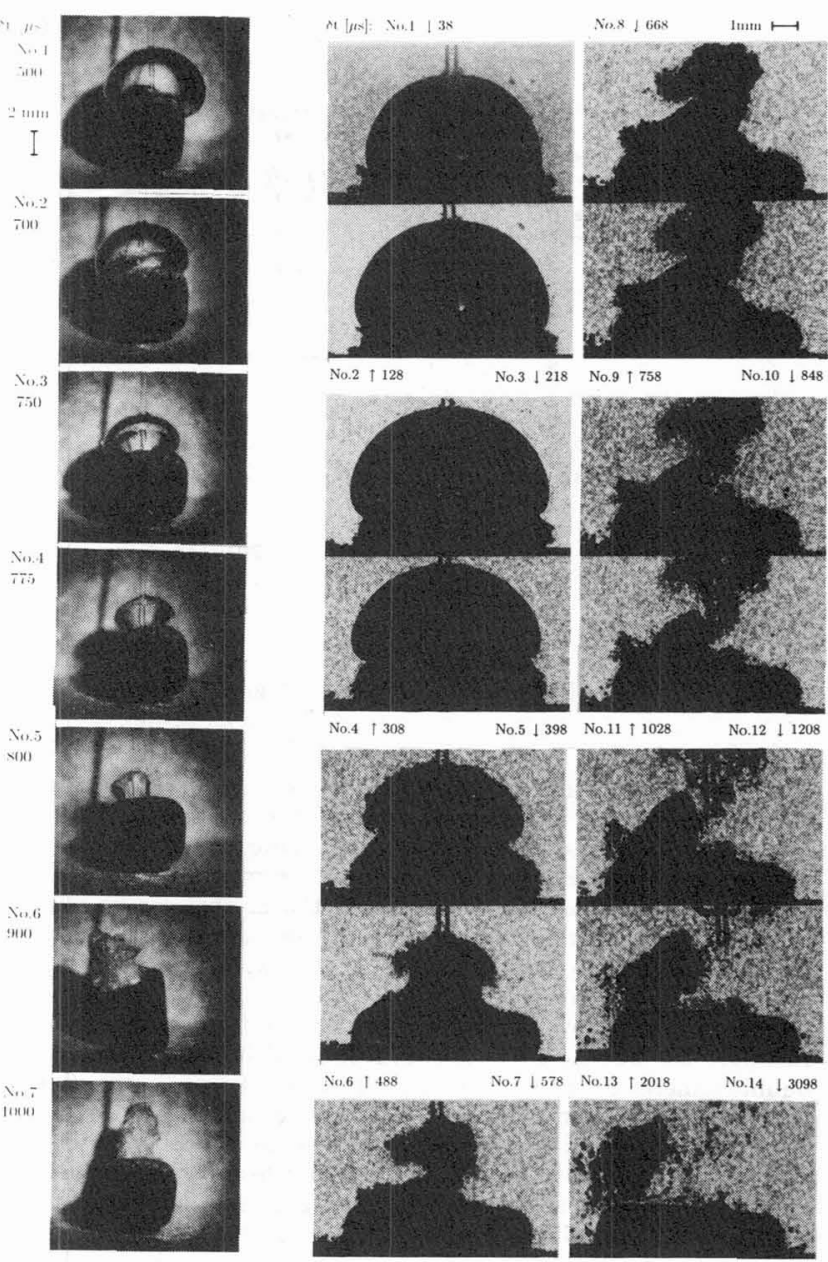

Series 2

arrangement of fig. 2
Series 3

arrangement of fig. 3

Fig.4 - Fragmentation of gallstones $-\mathrm{E}_{\text {fiber }}=150 \mathrm{~mJ}, \mathrm{~d}_{\text {fiber }}=400 \mu \mathrm{m}$

\section{References:}

[ 1 ] H. Schardin; Das Toeplersche Schlierenverfahren, VDI-Forschungsheft 367 (1934)

[ 2 ] M. Müller; Stoßwellenfokussierung in Wasser; Dissertation, RWTH Aachen (1987)

[ 3 ] A. Vogel, W. Lauterborn, R. Timm; Optical and acoustic investigations of the dynamics of laserproduced cavitation bubbles near a solid boundary; J. Fluid Mech., vol.206, pp.299-338 (1989)

[ 4 ] N.S. Nishioka, P. Teng, T.F. Deutsch, R.R. Anderson; Mechanism of laser-induced fragmentation of urinary and biliary calculy; Lasers in the Sciences 1(3),pp.231-245 (1987)

[ 5 ] C. Naudé and A. Ellis; On the mechanism of cavitation damage by nonhemisherical cavities collapsing in contact with a solid Doundary; J. of Basic Eingmeering, pp.648-656 (dec. 1961)

[6] M. Plesset, R. Chapman; Collapse of an initially spherical vapour cavity in the neighbourhood of a solid boundary; J. Fluid Mech. (1971), vol.47, part 2, pp. 283-290

[ 7 ] R. Blake, B. Taib, G. Doherty; Transient cavities near boundaries. Part 1. Rigid boundary; J. Fluid Mech. (1986), vol.170, pp.479-497

[ 8 ] J.W. Rayleigh; On the pressure developed in a liquid during the collapse of a spherical cavity; Philos. Mag.34, pp.94-98 (1917) 